\title{
Dynamic Behaviour of ISFET-based Sensor-Actuator Systems
}

\author{
W. OLTHUIS, J. LUO, B. H. VAN DER SCHOOT, J. G. BOMER and P. BERGVELD \\ Department of Electrical Engineering, University of Twente, P.O. Box 217, 7500 AE Enschede (The Netherlands)
}

\begin{abstract}
Rapid acid-base titrations can be performed at the surface of a noble-metal electrode with coulometrically generated ions. An ISFET is used as an indicator electrode to detect the equivalence point in the resulting titration curve. The time needed to reach the equivalence point is typically 0.5 to $10 \mathrm{~s}$ for acid/base concentrations ranging from $0.5 \times 10^{-3}$ to $20 \times 10^{-3} \mathrm{~mol} \mathrm{l}^{-1}$.

A model is presented describing the concentration profiles which appear during the coulometric generation of ions. The result of this model is in good agreement with corresponding measurements. These measurements are carried out with two different actuator electrodes, of which the processing steps are described.
\end{abstract}

\section{Introduction}

The chemical environment near a noble-metal actuator electrode can be controlled locally by the coulometric generation of $\mathrm{H}^{+}$or $\mathrm{OH}^{-}$ions at the electrode surface.

The ions thus generated are used as a titrant to perform an acid-base titration. The resulting concentration change is measured by a $\mathrm{pH}$-sensitive ISFET that is placed in close proximity to the actuator electrode. The rate of this change is related to the bulk concentrations of the buffering components in the solution [1].

The sensor-actuator device is schematically shown in Fig. 1.

The actual titration is performed within seconds in a sub-microliter volume formed by the actuator electrode surface in contact with the solution and the diffusion layer thickness. The $\mathrm{pH}$ change during the coulometic titration as measured by the ISFET is the result of diffusion mass transfer.

\section{Theory of Operation}

For the description of the operational mechanism, some assumptions are made. Mass transfer as a result of migration effects can be avoided by a sufficiently large excess of supporting electrolyte concentration and is therefore neglected here. Convective transfer in a non-stirred solution is also considered to be absent. Furthermore, only one-dimensional semi-infinite diffusion is taken into account, while the actuator electrode is considered to be an infinite planar electrode.

Because of technological limitations, the sensor and the actuator cannot be located at the same position. For the model given here, the twodimensional reality is simplified to a one-dimensional description. The actuator electrode of the sensor-actuator device is considered to be placed at $x=0$ and the sensor at $x=x_{\mathrm{s}}$ ( $x$-direction perpendicular to the planar actuator electrode). The device is placed in a non-stirred aqueous solution of acid HA. The several steps that can be distinguished after a cathodic current pulse is applied to the actuator electrode and the generation of $\mathrm{OH}^{-}$ions starts, are shown in Fig. 2(a)-(f), where the linearized concentration profiles $C_{\mathrm{HA}}(x, t)$ and $C_{\mathrm{OH}}(x, t)$ at six consecutive moments are given.

The moment that the actuator current is switched on at $t_{1}$, the concentration in the solution is still homogeneous and equals $C_{\mathrm{HA} \text {, bulk }}$ $\left[\mathrm{mol} \mathrm{m}^{-3}\right]$. The fast dissociation of $\mathrm{HA}$ and the rapidly established equilibrium of water result in a depletion of $\mathrm{HA}$ at the actuator electrode at $t_{2}$. At $t_{3}$ the HA concentration just reaches zero at the actuator electrode at $x=0$. The continuing generation of $\mathrm{OH}^{-}$ions at the actuator electrode now gives rise to the formation and increase of a concentration profile of $\mathrm{OH}^{-}$ions at $t_{4}$. This increase causes the reaction plane $\lambda(t)$, where the $\mathrm{HA}$ and the $\mathrm{OH}^{-}$concentration profiles meet, to move into the bulk solution. At $t_{5}$ this reaction plane, representing the plane where the equivalence point in the coulometric acid-base titration is reached, just passes $x_{\mathrm{s}}$ where the sensor of the device is considered to be placed. At $t_{6}$ the reaction plane $\lambda(t)$ diffuses further into the solution and the sensor now senses an alkaline solution instead of the original acid solution.

The $\mathrm{pH}$ changes detected by the sensor at $x_{\mathrm{s}}$ at the consecutive moments $t_{1}$ to $t_{6}$ result in a titration curve as given in Fig. $2(\mathrm{~g})$.

(C) Elsevier Sequoia/Printed in The Netherlands 


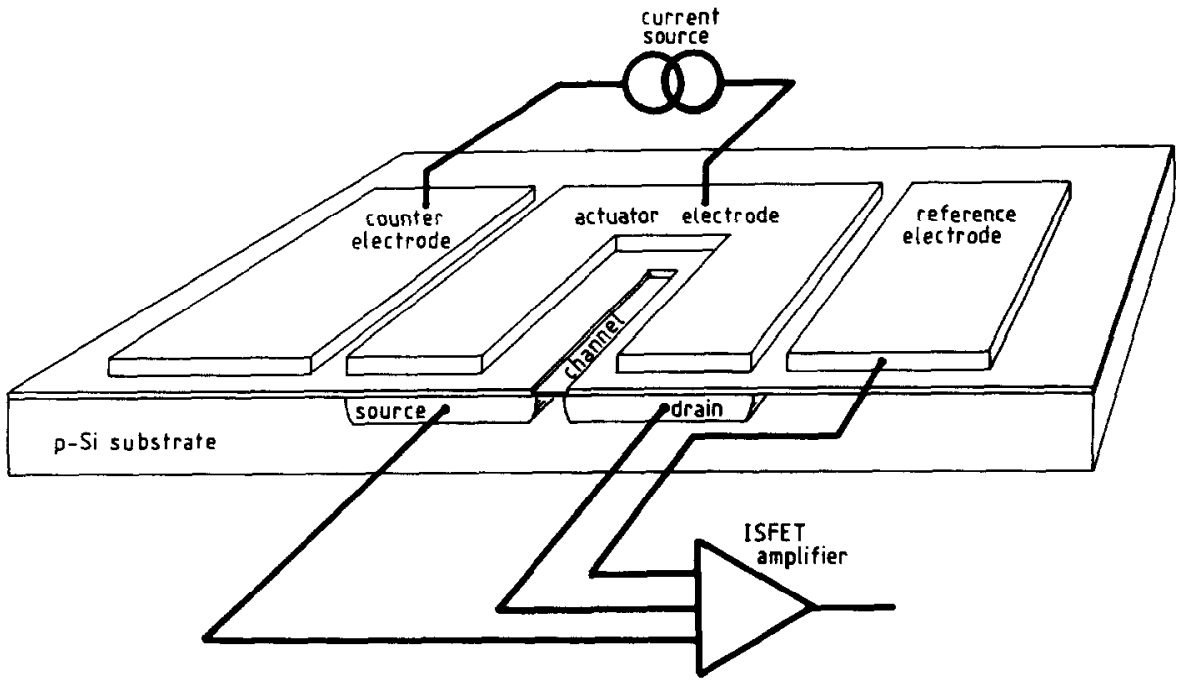

Fig. 1. Basic elements of the acid/base concentration sensor.

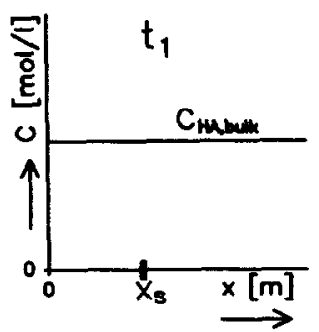

(a)

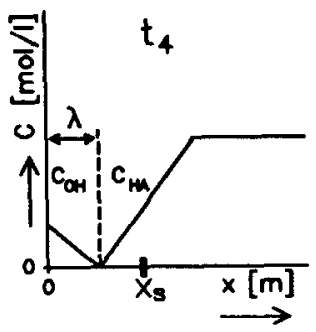

(d)

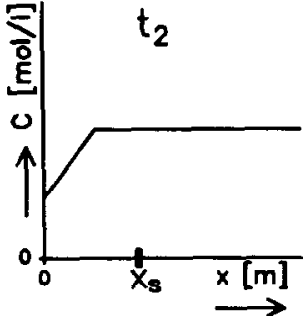

(b)

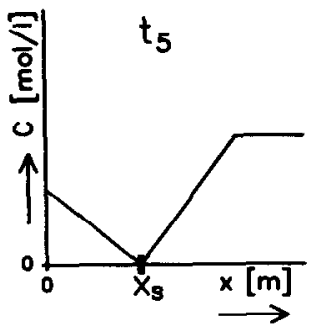

(e)

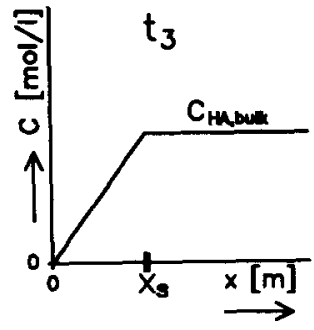

(c)

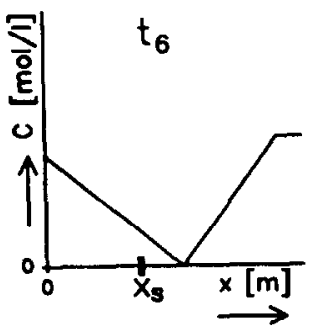

(1)

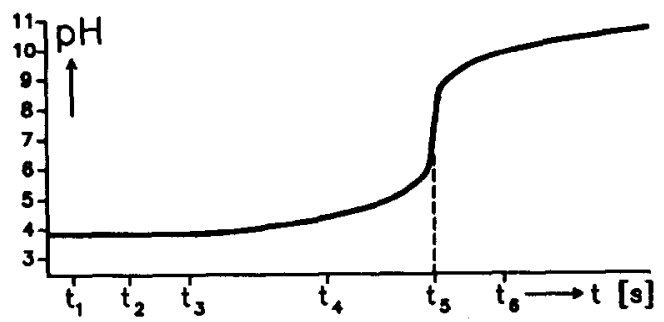

(g)

Fig. 2. (a)-(f) Linearized concentration profiles at the actuator electrode at six consecutive moments. (g) The pH change as sensed by the ISFET at $x_{\mathbf{s}}$, resulting in the titration curve as shown. 
It can be seen from Fig. 2 that the time $t_{\text {eq }}$ $\left(=t_{5}\right)$ needed to measure the equivalence point in the titration curve is the sum of two terms; the first term is the time $t_{\text {Sand }}\left(=t_{3}\right)$ needed to totally deplete the HA concentration at $x=0$, and the second term $t_{\text {delay }}\left(=t_{5}-t_{3}\right)$ describes the time needed for the reaction plane $\lambda(t)$ to reach the position of the sensor $x_{\mathrm{s}}$

$t_{\text {eq }}=t_{\text {Sand }}+t_{\text {delay }}$

The well-known Sand equation [2], from which $t_{\text {Sand }}$ is obtained reads as follows

$t_{\text {Sand }}=\left\{\frac{C_{\mathrm{HA} . \text { bulk }} F \sqrt{\pi D_{\mathrm{HA}}}}{2 j_{\mathrm{c}}}\right\}^{2}$

where $D_{\mathrm{HA}}\left[\mathrm{m}^{2} \mathrm{~s}^{-1}\right]$ is the diffusion coefficient of acid $\mathrm{HA}, F\left[\mathrm{C} \mathrm{mol}^{-1}\right]$ is Faraday's constant, and $j_{\mathrm{c}}\left[\mathrm{A} \mathrm{m}^{-2}\right]$ the current density.

Then, for $l>t_{\text {Sand }}$ the concentration profiles become as shown in Fig. 2(c)-(f). The reaction plane moves into the solution over distance $\lambda(t)$. The equivalence point in the titration curve is detected when $\lambda(t)$ reaches $x_{s}$, the position of the sensor.

The time $t_{\text {delay }}$ this takes can be calculated by considering the flux and the amount of generated and depleted ions in both the film with the $\mathrm{OH}^{-}$ gradient and the film with the HA gradient [3]. For the assumed linear profiles, this calculation results in

$t_{\text {delay }}=\frac{(\pi-2) x_{\mathrm{s}}^{2}}{2 \pi D_{\mathrm{OH}}}+\frac{F C_{\mathrm{HA}, \text { bulk }} x_{\mathrm{s}}}{j_{\mathrm{c}}}$

where $D_{\mathrm{OH}}\left[\mathrm{m}^{2} \mathrm{~s}^{-1}\right]$ is the diffusion cocfficient of $\mathrm{OH}^{-}$ions.

\section{Experiments and Results}

The $\mathrm{pH}$-sensitive ISFET is fabricated following the usual NMOS processing steps [4]. The actuator electrode consists of a thin $(0.6 \mu \mathrm{m}) \mathrm{Au}$ film evaporated around the gate on top of an $\mathrm{Ag}$ film $(0.1 \mu \mathrm{m})$ and a $20 \mathrm{~nm}$ Ti film for protection of the ISFET against mobile $\mathrm{Au}$ atoms and good adhesion of the film on the underlying oxide, respectively.

The titanium and the silver film are evaporated in one cycle and patterned using $0.15 \mathrm{M} \mathrm{Fe}\left(\mathrm{NO}_{3}\right)_{3}$ for the etching of silver and HF (1:20 in water) as etchant for titanium. After the evaporation of gold on top of the silver film, this layer is etched in a solution containing $I_{2}$ and $K I$ ( 1 and $30 \mathrm{~g} / \mathrm{l}$ respectively). The result is an actuator electrode shape as shown in Fig. 3. A layer of polyimide determines the active area $\left(1 \mathrm{~mm}^{2}\right)$ of the actuator electrode in contact with the solution.

The chip $(3 \times 4 \mathrm{~mm})$ is glued on a piece of printed circuit board $(1 \times 10 \mathrm{~cm})$ together with a

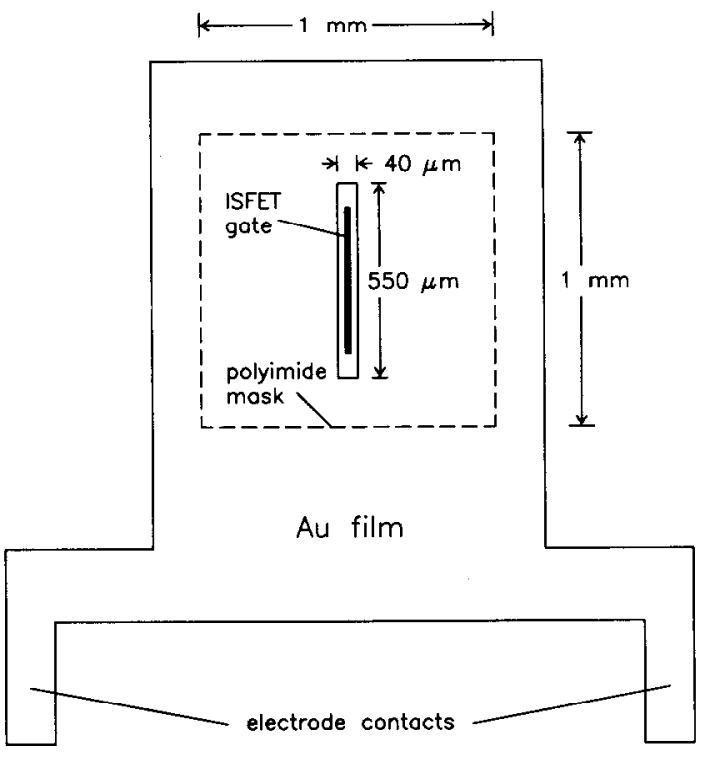

Fig. 3. Top view of the actuator electrode.

second chip. The bonding wires and the $\mathrm{Cu}$ connection strips are protected by synthetic resin. A thin $\mathrm{Au}$ film evaporated on a $\mathrm{Cu}$ strip on the reverse side of the printed circuit board serves as a counter electrode for the supplied actuator current.

The measurement set-up is shown in Fig. 4.

The ISFET of the second chip can be used as a reference, because the bulk $\mathrm{pH}$ does not change during the experiment. The Au 'actuator' electrode shaped around the reference ISFET serves as pseudo reference electrode to ground the solution. A differential measurement of both ISFET output signals results in the desired signal. The equivalence point in the titration curve is calculated by the maximum of its first derivative.

A series of measurements is carried out to determine $t_{\mathrm{eq}}$ in solutions with different acetic acid concentrations. The result is presented in Fig. 5, curve 1 .

It is clear from Fig. 5, curve 1, that the square root of $t_{\mathrm{eq}}$ depends linearly on the acid concentra-

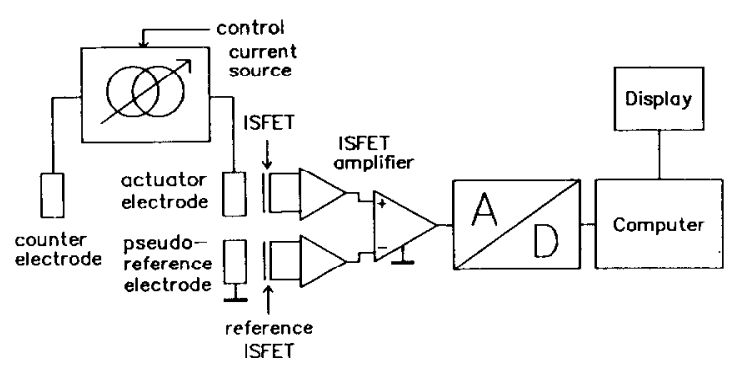

Fig. 4. Measurement set-up. 


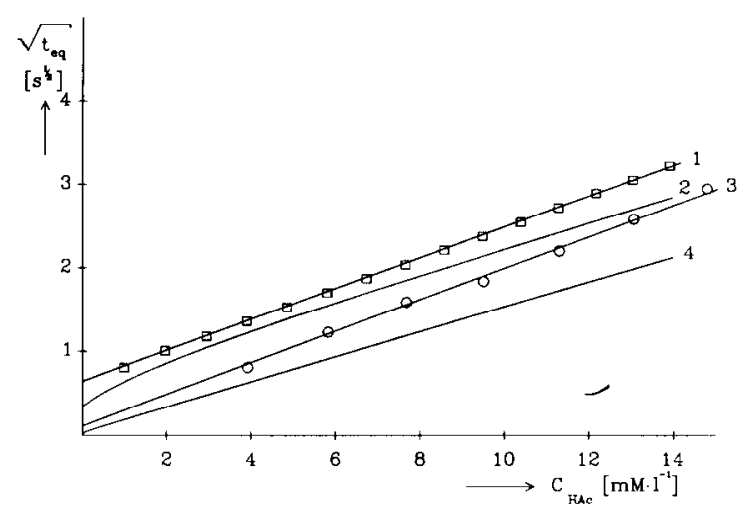

Fig. 5. Results of coulometric titration of HAc; $j_{\mathrm{c}}=20$ $\mathrm{A} \mathrm{m}^{-2}$. Actuator electrode shaped around the gate: curve 1, measurement results; curve 2, results of the model. Bridgetype actuator electrode: curve 3 , measurement results; curve 4 , results of the model. $D_{\mathrm{HAc}}=1.21 \times 10^{-9}$ and $D_{\mathrm{OH}}=$ $5.27 \times 10^{-9} \mathrm{~m}^{-2} \mathrm{~s}$.

tion. This is in agreement with the results of the model (curve 2) of eqns. (1), (2) and (3), although the calculated slope is approx. $10 \%$ smaller than the measured slope. This is possibly caused by the fact that the model only includes the diffusion of undissociated HAc molecules instead of a mixture of fast $\mathrm{H}^{+}$ions and slow HAc molecules.

The result of the model, curve 2 , is linearly extrapolated to intersect the vertical axis at the same point as the result of the corresponding measurement (curve 1). The value for the position of the sensor $x_{\mathrm{s}}$ with respect to the actuator was thus estimate at $x_{\mathrm{s}}=50 \mu \mathrm{m}$. It is not fully realistic to compare this value with the real distance of approx. $20 \mu \mathrm{m}$, because the model description is only one dimensional.

The relatively large delay time, caused by the distance between the actuator electrode and the sensor, can be reduced drastically, according to the model (eqn. (3)), when this distance is decreased. Therefore, a second type of actuator electrode is developed. This electrode consists of 27 $\mathrm{Au}$ bridges of $10 \mu \mathrm{m}$ width over the ISFET gate, spaced $10 \mu \mathrm{m}$ apart. The bridge-to-gate distance is approx. $1 \mu \mathrm{m}$.

The processing starts with the evaporation of $20 \mathrm{~nm}$ titanium and $500 \mathrm{~nm}$ silver on top of the ISFETs. To free the gate area, the silver is etched with $0.15 \mathrm{M} \mathrm{Fe}\left(\mathrm{NO}_{3}\right)_{3}$ and the titanium with $\mathrm{HF}$ (1:20 in water). Then a spacer is applied on the gate area by patterning a spun resist layer (3500 r.p.m., $20 \mathrm{~s}$ ) that is composed of positive photoresist (AZ 1450J) and ethylglycolacetate (3:1). After patterning, the resist is cured for $0.5 \mathrm{~h}$ at $160^{\circ} \mathrm{C}$ in a $\mathrm{N}_{2}$ atmosphere. The next step is the spinning and patterning of a lift-off mask. After curing at $90^{\circ} \mathrm{C}$ for $20 \mathrm{~min}$, this layer of positive photoresist is dipped for $5 \mathrm{~min}$ in chlorobenzene

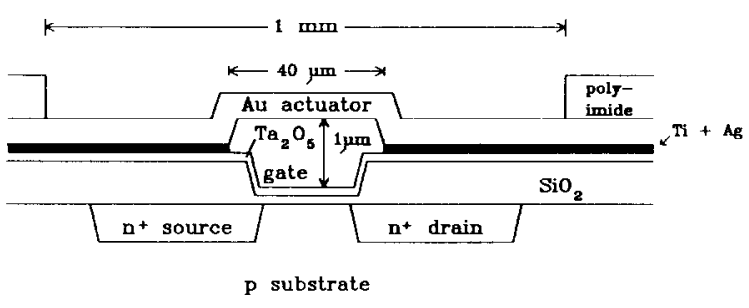

Fig. 6. Cross section of the bridge-type sensor-actuator device.

to harden the top layer in order to facilitate the process of lift-off. Then the Au film $(0.6 \mu \mathrm{m})$ is evaporated on top of the Ti/Ag film, the spacer and the lift-off mask. The lift-off mask is dissolved using acetone, whereafter the spacer is removed with positive resist stripper (Baker PRS 1000) at $80^{\circ} \mathrm{C}$. The cross section of the device with bridgetype actuator is shown in Fig. 6. A SEM photo (Fig. 7) shows the bridges.

The results of measurements carried out with this device are presented in Fig. 5, curve 3. The slope of this measured curve is equal to the slope of the measured curve 1 from the device with the flat actuator electrode; the difference in distance between the sensor and actuator of the two devices does not influence the sensitivity. This is expected from the result of the model (curves 2 and 4). The decrease of the delay time that is now expected from the model is clearly noticeable by the shift of the measured curve 3 towards zero in the absence of any acid. The result of the model, curve 4 , is linearly extrapolated to intersect the vertical axis at the same point as the result of the corresponding measurement, curve 3 . The position of the sensor was thus estimated at $x_{\mathrm{s}}=1.0 \mu \mathrm{m}$, which is in close agreement with the real distance of approx. $1 \mu \mathrm{m}$.

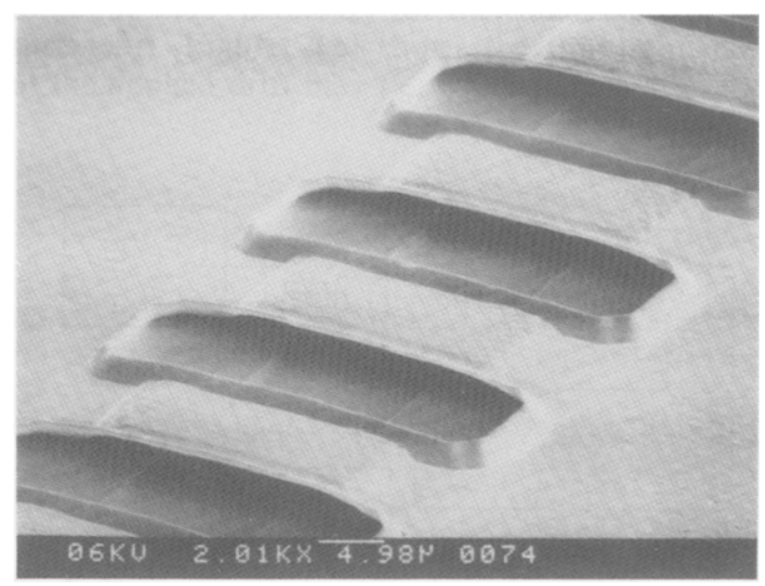

Fig. 7. SEM photo of the bridge-type actuator electrode. 


\section{Conclusions}

The model of the acid-base concentration sensor, that describes the dynamic behaviour of the concentration profiles at the actuator electrode, including the non-zero distance between the sensor and actuator, is in good agreement with corresponding measurements.

\section{Acknowledgements}

B.H.v.d.S thanks the Royal Netherlands Academy of Arts and Sciences for the award of a fellowship. This investigation is part of the research program of the Foundation for Funda- mental Research on Matter (FOM), which is supported by the Netherlands Technology Foundation (STW).

\section{References}

1 W. Olthuis, B. H. van der Schoot, F. Chavez and P. Bergveld, A dipstick sensor for coulometric acid-base titrations, Sensors and Actuators, 17 (1989) 279-283.

2 A. J. Bard and L. R. Faulkner, Electrochemical Methods, Fundamentals and Applications, Wiley, New York, 1980.

3 W. Olthuis, J. Luo, B. H. van der Schoot, P. Bergveld, M. Bos and W. E. van der Linden, Modeling of non-steady state concentration profiles at ISFET-based coulometric sensor-actuator systems, Anal. Chim. Acta, to be published.

$4 \mathrm{~T}$. Matsuo and M. Esashi, Methods of ISFET fabrication, Sensors and Actuators, 1 (1981) 77-96. 